DE DE GRUYTER

OPEN

G

BULGARIAN ACADEMY OF SCIENCES

CYBERNETICS AND INFORMATION TECHNOLOGIES • Volume 14, No 2

Sofia $\bullet 2014$

Print ISSN: 1311-9702; Online ISSN: 1314-4081

DOI: $10.2478 /$ cait-2014-0019

\title{
Efficient Data Transfer by Mobility Adjustment Algorithm for Clustered Mobile Ad-Hoc Networks
}

\author{
I. Alagiri*, V. Madhuviswanatham**, P. Venkata Krishna** \\ ${ }^{*}$ School of Information Technology and Engineering,VIT University, Vellore-14 \\ ${ }^{* *}$ School of Computing Science and Engineering, VIT University, Vellore-14 \\ Emails:ialagiri@vit.ac.invvmadhuviswanatham@vit.ac.in pvenkatakrishna@vit.ac.in
}

\begin{abstract}
Mobile Ad-hoc NETwork (MANET) is a prevalent deployable network for easy plug-in and it is widely applied for many real time scenarios. Clustering is a well known solution for efficient communication among nodes with least control overheads. The communication link breaks between nodes when a node moves beyond the transmission range of another node because of mobility. Frequent link breaks happen because of nodes mobility which cannot be controlled without increasing the network control overheads. The authors propose an Mobility Adjustment Routing (MAR) routing algorithm for establishing a stable path between the source and the destination. In this approach the choice of cluster heads based on the smallest weight age, node mobility and remaining battery power are used as a metric for weight computation. The cluster head and the gate way nodes forward $R R E Q$ packets to set up a path between the source and the destination which proves efficient communication, before forwarding a RREQ packet cluster the head/gateway node compares its mobility value with RREQ and updates the least value in RREQ. The destination node advertises the least mobility value to the remaining nodes in the path with the help of RREP packet, therefore stable paths are found without increasing the network control overheads. The simulation results done with the help of network simulator 2 show that the algorithm proposed performs well even at higher traffic load compared to existing algorithms.
\end{abstract}

Keywords: MANET, MAR, WCA, WBCA, GDMAC, LID. 


\section{Introduction}

Mobile nodes collectively form a Mobile Ad-hoc NETwork (MANET), which communicate over radio. MANET's are very flexible networks and do not need any central administrator or an existing infrastructure for communication. They transmit data directly to the nodes that are in their transmission region out of the region nodes reached with the help of intermediate nodes. The nodes not only behave like a host, they are capable to send packets to other nodes with the help of intermediate nodes, therefore this type of networks are also called multi-hop ad hoc networks [1]. The topology is dynamically changed due to nodes mobility which affects the data transfer rate. Designing an efficient on demand routing protocol for delivering packets to the destination is a crucial issue in MANET.

Finding a route between the end points is the main problem in MANET because of nodes mobility. Different approaches are available in literature that handle this problem, but none of them fits for all the cases.

In this paper we present a new approach - Mobility Adjustment Routing (MAR) to handle the node mobility without increasing the control overheads. The least mobility value was found during the route discovery process and updated during the route replay to increase the life time of a path.

The rest of the paper is organized as follows. In Section 2 we present the basics and the work done in similar areas. In Section 3 we discuss the weight calculation for cluster heads and cluster formation. In Section 4 we present the proposed algorithm in details. Section 5 discusses the stability of MAR protocol. Subsequently, in Section 6 performance analyses through simulations are presented. Finally Section 7 concludes the paper.

\section{Basics and related works}

\subsection{Basics}

Several routing protocols have been proposed [2-7], based on the routing information update mechanisms [8]. The routing protocols are broadly classified as proactive (Table Driven), reactive (On demand) and hybrid routing.

Proactive routing protocols, such as OLSR [3], DSDV [2] maintain up-to-date routing information in the form of a table. The node mobility causes frequent topology changes in ad hoc networks. These changes are often updated in the routing table to keep the up-to-date topology information, which increases control overheads; therefore proactive routing protocols are not suitable.

On-demand routing protocols, such as AODV [4] and DSR [5] discover the route whenever needed, they discover the route by flooding a RREQ packet to all neighbours. Each neighbour node blindly broadcasts RREQ until it reaches the destination which leads to a broadcast storm problem. If $N$ nodes are present in the network, then the number of broadcast packets is equal to $N-2$.

Features of both proactive and reactive protocol are merged to form the hybrid routing protocols [6-8] which use a proactive approach within the zone and ondemand approach across the zone. 
Clustering controls the flooding of RREQ packets, therefore it reduces the control overheads. Clustering is the process of grouping the nodes into small groups, each group consisting of a cluster head, cluster member and gateway nodes. The cluster heads and gateway nodes allowed to send RREQ packets to the rest of the nodes, can listen to RREQ packet but they cannot send it to other nodes.

\subsection{Related works}

The concept of clustering initially proposed in [9], many clustering algorithms are found in literature and most of them follow the greedy approach. The minimum number of cluster heads [10-13] reduces the end-to-end delay and congestion and the cluster stability is improved by minimizing the cluster head election process $[14$, 15]. The node having the lowest ID [14] is selected as a cluster head and will keep its place until none of the nodes having the lowest cluster ID when compared to it leads to a faster energy drain in the cluster head and reduces the network life time.

The cluster head is selected based on the degree of connectivity [10] to its neighbours. If the degree of connectivity is high, then the node is selected as a cluster head. Topology changes of the ad hoc network leads to the cluster head selection.

The mobility metric based algorithm [16] proposes the node, having the least mobility to be selected as a cluster head which improves the cluster stability and network stability, the process of calculating the relative mobility for each node degrades the network performance.

Distributed Mobility Adaptive algorithm [17] eliminates the problem of nonmobility of the host during cluster setup and maintenance. When two cluster heads are within the transmission region of each other, the cluster head with the lowest weight is forced to resign its role and it will act as a member to the other head that leads to reduction of the clustering efficiency.

In the Generalized Distributed Mobility Adaptive Clustering (GDMAC) algorithm [18] some neighbours heads are equal to ' $K$ '. The role of the neighbour cluster heads changes only when the weight difference between them exceeds a particular threshold value.

The Weighted Clustering Algorithm (WCA) [19] uses different node limits to compute the weight which plays a major role in the cluster head choice. Huge information is obtained from the nodes to calculate the weight reducing the energy level of every node.

Local Information No Topology (LINT) algorithm [20] is based on the degree of connectivity, transmission range adjusted to keep the neighbours within a particular region. Ad hoc network topology changes leads rapidly to frequent changes in the cluster head.

Self adjusting transmission range control protocol [21] maintains the predefined number of nodes as neighbours to keep up the neighborhood relationship.

Topology Control by Transmission Range Adjustment [22] uses the mobility factor and the remaining battery power as metrics to compute the node weight. The node having more weight age is selected as a cluster head mobility factor adjusted 
by analyzing the average mobility at a particular time interval. Getting the proper mobility factor is a difficult task, since mobility is not constant.

All these proposals discuss minimizing the cluster head selection process, maintaining the same number of neighbours to control topology. The transmission range adjustment has left some nodes unreachable, so the data transfer was not possible to such nodes. In our paper this problem was overcome by adjusting mobility to form a stable path.

\section{Clustering algorithm}

A graph $G=(V, E)$ is used to model the ad hoc network in which $V, E$ is a finite set of nodes and bidirectional edges that connect the nodes. Cardinality defined as the number of elements in a particular set. The cardinality of set $V$ is constant, but the cardinality of set $E$ is not constant, since it depends on the nodes mobility. Each node $v_{i} \in v$ must have unique identity, mobility $v_{\text {mob }}$, and the largest transmission range $v_{\text {tr }}$. The node $v_{i}$ is within the transmission range of $v_{j}$ if dist $\left(v_{i}, v_{j}\right)<v_{\text {tr }}$.

\subsection{Features of the clustering algorithm}

1. The mobility of the nodes is increased or decreased as per requirements, but a node can have maximum mobility of $v_{\max }$.

2. The weight of each node is calculated using the mobility and the remaining battery power.

3. At the first stage a volunteer cluster head choice function is called.

4. The energy drain rate of the cluster heads was very high. The cluster head re-election process is called when the battery level of the cluster head falls below a threshold value in order to keep up balanced energy level in all the nodes.

\subsection{Neighbor identification}

Before forming the cluster it is necessary to identify one hop nodes in this algorithm. One hop node is identified by exchanging Cluster Head Identification REQuest (CHIREQ), the packet format is shown in Fig. 1. Initially all the status field is set to false or 0 . The node that receives the CHIREQ packet, sends a Neighbour REPlay (NREP). NREP packet format is shown in Fig. 2. The node that receives the CHIREQ packet, sends a NREP, after receiving NREP packet Cluster Head Advertise Packet (CHAP) is sent to its neighbour. The packet format of CHAP is shown in Fig. 3. Once CHAP reaches the one hop nodes, they will update their status fields. Three different fields are used to denote the node status:

CH (Cluster Head),

CM (Cluster Member),

CG (Cluster Gateway).

The node elected as a cluster head will update its $\mathrm{CH}$ status to true or 1 . If a cluster member receives more than one CHAP, it will set its CG status field as 1 .

\begin{tabular}{|l|l|l|l|l|}
\hline Source ID & \multirow{2}{*}{ Weight } & \multicolumn{3}{|c|}{ Status } \\
\cline { 3 - 5 } & & $\mathrm{CH}$ & $\mathrm{CM}$ & $\mathrm{CG}$ \\
\hline
\end{tabular}

Fig. 1. CHIREQ packet format 
The node weight calculated is as follows

$$
\text { Weight }_{i}=M_{i}+\frac{1}{\mathrm{RB}_{i}},
$$

where:

Weight $_{i}$ is weight of $i$-th node.

$M_{i} \quad$ is mobility of $i$-th node,

$\mathrm{RB}_{i} \quad$ is Remaining Battery power of $i$-th node,

The node remaining battery power calculated is as follows:

where:

$$
\mathrm{RB}=\mathrm{BP}_{\max }-\mathrm{BP}_{\text {cons }} \text {, }
$$

$\mathrm{BP}_{\max }$ is maximum battery power,

$\mathrm{BP}_{\text {cons }}$ is consumed battery power.

\begin{tabular}{|l|l|l|l|l|l|}
\hline \multirow{2}{*}{ Source ID } & \multirow{2}{*}{ Destination ID } & \multirow{2}{*}{ Weight } & \multicolumn{4}{|l|}{ Status } & \\
\cline { 4 - 5 } & & & CH & CM & CG \\
\hline
\end{tabular}

Fig. 2. Neighbour replay packet format

\begin{tabular}{|l|l|l|l|}
\hline Source ID & Cluster ID & Weight & Neighbors list \\
\hline
\end{tabular}

Fig. 3. CHAP packet format

\subsection{Steps associated with neighbour identification}

Step 1. Node S sends CHIREQ packets to all nodes that are in its transmission region.

Step 2. Node D which receives CHIREQ packet, replays with NREP. This packet has the details about node D piggybacked into it.

Step 3. After receiving all NREP packets, the node with higher weight is selected as a cluster head and advertised to its one hop nodes.

Step 4. The cluster head updates its neighbouring table.

Step 5. The member node receives a request from another cluster, then it updates its status as a gateway node and informs its cluster head.

\begin{tabular}{|c|c|c|}
\hline Cluster ID & Members ID & Gateways ID \\
\hline
\end{tabular}

Fig. 4. Cluster table

The cluster table is used by $\mathrm{CH}$ to store its member information and the gateways information format of the cluster table is shown in Fig. 4.

A node which is a member in a cluster receives cluster advertisement information from another node; then it will update its status to the gateway and inform the cluster head, each gateway node maintains the list of cluster ID to send any request. The gateway table maintained by the gateway node is shown in Fig. 5.

\begin{tabular}{|l|l|}
\hline Gateway ID & Clusters ID \\
\hline
\end{tabular}

Fig. 5. Gateway table 


\section{MAR}

MAR uses hierarchical network topology to organize the nodes in terms of clusters. A path between node $x$ to node $y$ is in the form of $x-\mathrm{CH}_{1}-\mathrm{CG}_{1}-\mathrm{CH}_{2}-\mathrm{CG}_{2}-\ldots$ $\mathrm{CH}_{i} \ldots \mathrm{CG}_{j}-y$, where $\mathrm{CH}_{i}$ and $\mathrm{CG}_{j}$ are $i$-th cluster head and $j$-th cluster gateway. A sample network topology with five nodes is shown in Fig. 6. The nodes were identified by unique numbers from 1 up to 5 . It is clear from Fig. 6 that node 5 lies within the transmission region of node 1 and 3, whereas nodes 2 and 4 are within the transmission region of nodes 1 and 3 . Node 5 will receive more than one CHAP packet, so it will act as a gateway node.

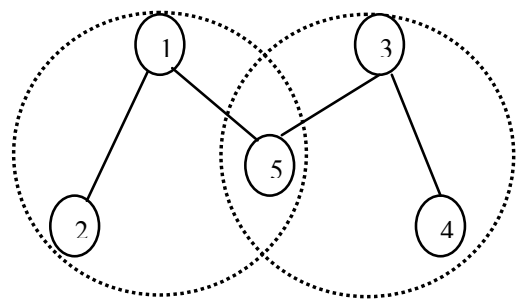

Fig. 6. Sample network

\subsection{Route discovery}

In the route discovery phase, when a node wants to communicate with another node, it sends RREQ packet to its cluster head. Fig. 7 shows the fields of RREP packet. If the destination is a member of the cluster head, it will send a RREP packet to the sender. Fig. 8 shows the fields of RREP packet. If the destination is not a member of the cluster head, it updates its mobility information in the mobility field of RREQ, since the cluster head mobility is always smaller than its member mobility. In general, when the node receiving RREQ is with smaller mobility compared to the sender, then the mobility field is updated by a new value. Fig. 7 shows RREQ fields of MAR.

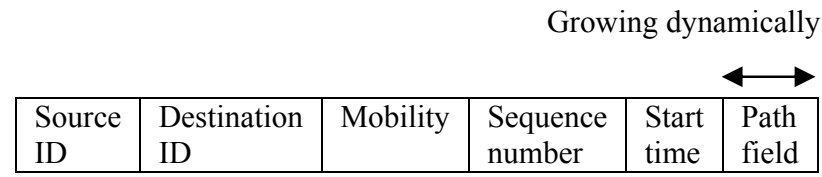

Fig. 7. RREQ packet

\subsection{Loop detection}

The path field in a RREQ packet consists of an address or ID of the nodes that are visited in the path from the source to the destination. When an intermediate node receives a RREQ, it first checks whether its address is already present in the path field or not. If it does not exist, its address is added to the path field forward to the next node, else the RREQ is discarded to avoid loops (Figs 8 and 9). 


\begin{tabular}{|l|l|l|l|l|}
\hline Source ID & Destination ID & $\begin{array}{l}\text { Start } \\
\text { Time }\end{array}$ & $\begin{array}{l}\text { Received } \\
\text { Path }\end{array}$ & Mobility \\
\hline
\end{tabular}

Fig. 8. RREP Packet structure

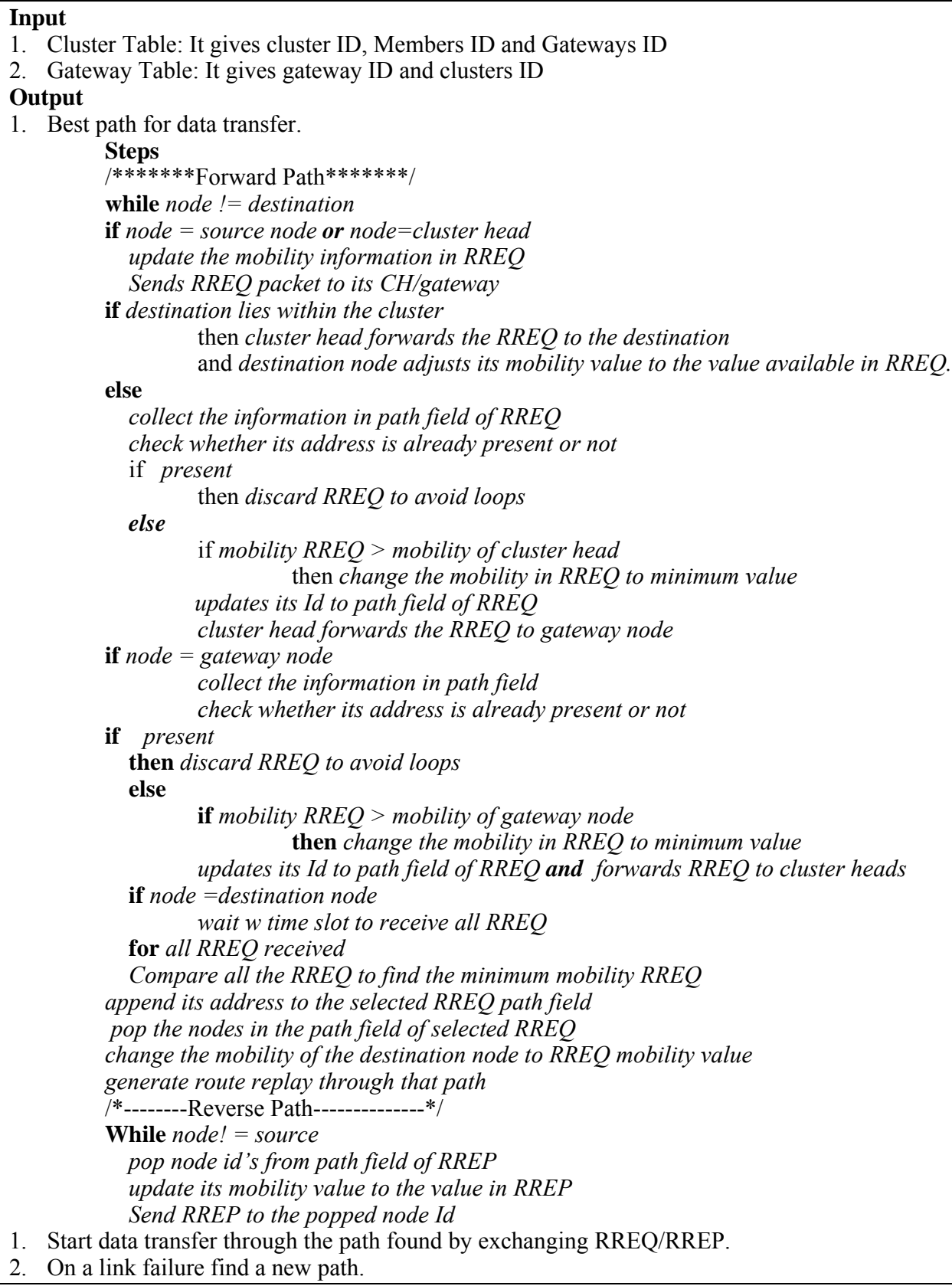

1. Start data transfer through the path found by exchanging RREQ/RREP.

2. On a link failure find a new path.

Fig. 9. MAR algorithm 


\section{Stability analysis}

The stability of the mobility model has been studied by many researchers [23-29]. In their papers they analyze the various events that occur at different layers of the network, but they never discuss the mobility model, most of the cases stability analysis depends both on mobility and network protocols. Random Way Point Mobility Model (RWPMM) [29] states that if Pointwise ergodic theorem is satisfied by a mobility model, then it is stable.

Definition 1 [29, Definition 4]. A mobility model which satisfies point wise ergodic theorem is stable.

The main result of the present paper states that the Ergodic theorem for node movement is inferred to derive the Ergodic theorem of route selection which follows from the theory of finite state source coders [30] and from Asymptotically Mean Stationary (AMS) [31, 32] random process.

\subsection{Preliminaries}

\subsubsection{Stable mobility model}

Consider a mobile network with $|v|$ mobile nodes $v=v_{1}, v_{2}, \ldots, v_{|v|}$ where each node moves randomly in a discrete finite state space $S$. The movement of each node, $v_{j}, j \in\{1,2,3, \ldots,|v|\}$, described by a discrete finite random process $\left\{s_{n, j}\right\}_{n=0}^{\infty}$, where $k$-th random variable $s_{k, j}$ represents the node $v_{j}$ 's location at time $k$. The location of each node in $S$ at time $k$ is represented by $X_{n}=\left(s_{k, 1}, s_{k, 2}, \ldots, s_{k,|v|}\right)$.

\subsection{2. $n$-fold Cartesian product}

$n$-fold Cartesian product of the sets $A_{1}, A_{2}, \ldots, A_{n}$ is the set of $n$-tuples $A_{1} \times A_{2} \times, \ldots, \times A_{n}=\left\{\left(a_{1}, a_{2}, \ldots, a_{n} \mid a_{i} \in A_{i} \forall 1 \leq i \leq n\right)\right\}$.

The discrete finite alphabet of $X_{n}$ is denoted by using $|v|$-fold Cartesian product $x=\sum_{i=1}^{|v|} s_{i} ; \quad s_{i}=s$.

The probabilistic description of the mobility model is given by $\left\{X_{n}\right\}_{n=0}^{\infty}$ using $n$-fold Cartesian product $x^{\infty}=\sum_{n}^{\infty} x_{i} ; \quad x_{i}=x$.

The sequence $X=x_{0}, x_{1}, x_{2}, \ldots$ represents a particular element in the space $\left\{X_{n}\right\}_{n=0}^{\infty}$ where $x_{i} \in X ; \quad \forall i=0,1,2 \ldots$ A stable mobility model is defined with the help of the bounded function $\psi_{x}$ which consists of every function used in the simulation.

Definition 2. A random process mobility model $\left\{X_{n}\right\}_{n=0}^{\infty}$ is stable if for every $x$ the set of probability 1 and for every $\psi_{x}$ if $f \in \psi_{x}$ exists the limit $\langle f\rangle(x)=\lim _{n \rightarrow \infty}\langle f\rangle_{N}(x)$. 
Lemma 1. A random process discrete mobility model $\left\{X_{n}\right\}_{n=0}^{\infty}$ is stable $\Leftrightarrow\left\{X_{n}\right\}_{n=0}^{\infty}$ is AMS.

\subsection{Network topology model}

Ad-hoc network viewed by directed graph nodes in the network considered as vertices and the link connecting the nodes are considered as edges in the graph.

Definition 3. Consider a mobile network with $|v|$ mobile nodes $v=v_{1}, v_{2}, \ldots, v_{|v|}$ node movement described by a random process mobility model $\left\{X_{n}\right\}_{n=0}^{\infty}$. Space $X_{n}=\left(S_{n, 1}, \ldots, S_{n,|v|}\right)$ represents the location of nodes $v_{i} \in v$ at time $n$ for each pair of points $\left(s, s^{\prime}\right) \in S$ a bounded function $E$ is defined as $E: S \times S \rightarrow C$, where $C$ is the cost or capacity. The network topology at time $n$ is defined by $|v| \times|v|$ matrix

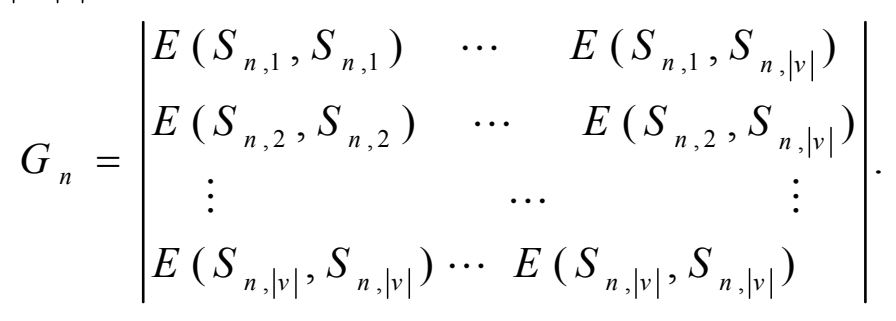

Let $G$ denotes the alphabet in $G_{n}$.

A link between two nodes exists if the distance between them is less than the transmission region

$$
E\left(s, s^{\prime}\right)=\left\{\begin{array}{rr}
1 \quad \text { if } d\left(s, s^{\prime}\right) \leq R \\
0 \quad \text { otherwise }
\end{array}\right.
$$

Lemma 2. Consider a random process mobility $\left\{X_{n}\right\}_{n=0}^{\infty}$ with topology $G_{n}$ at times $n=0,1,2, \ldots$ defined by Definition 3 . If the mobility model is stable, then $\left\{X_{n}\right\}_{n=0}^{\infty}$ and $\left\{G_{n}\right\}_{n=0}^{\infty}$ are AMS.

\subsection{Assumptions summary}

A source node $v_{i}$ wants to send data to sink node $v_{j}$ at time units $k=0,1,2, \ldots$ using MAR protocol. Let set $\mathfrak{R}$ denotes a collection of connected and disconnected links which form the route between $v_{i}$ and $v_{j}$. The route selected by MAR is given by $r_{k} \in \mathfrak{R}$ at time $k$. Three different functions are modeled to describe the route discovery, the route maintenance and mobility setup. These functions are $D: G \rightarrow \mathfrak{R}, M: G \times \mathfrak{R} \rightarrow\{1,0\}$ and $U: \mathfrak{R}_{s} \rightarrow \operatorname{Min}\left\{\operatorname{Mob}\left(v_{i}\right)\right\}$. 


\subsection{Stable route selection}

Definition 4. The root selection random process $\left\{R_{n}\right\}_{n=0}^{\infty}$ is stable if it satisfies the point wise ergodic theorem. Specially $\left\{R_{n}\right\}_{n=0}^{\infty}$ is stable if it exist a subset of sequence $\hat{R}^{\infty} \subseteq R^{\infty}$ with probability 1 , such that $\forall r \in \hat{R}^{\infty}$, the following limit exists

$$
\langle f\rangle(r)=\lim _{N \rightarrow \infty} \frac{1}{N} \sum_{n=0}^{N-1} f\left(r_{n}, r_{n+1}, r_{n+2}, \ldots\right) \forall \psi(f) \text { on } R^{\infty} .
$$

Definition 5. The routing protocol is stable if for every stable mobility model, the selected random process $\left\{R_{n}\right\}_{n=0}^{\infty}$ is stable.

The main result is following

Theorem 1. MAR described in Section 3 with a topology model defined by Definition 3, is stable.

P r o o f: The mobility model $\left\{X_{n}\right\}_{n=0}^{\infty}$ is said to be stable by Lemma 2, $\left\{X_{n}\right\}_{n=0}^{\infty \infty}$ and $\left\{G_{n}\right\}_{n=0}^{\infty}$ are AMS.

MAR protocol is described by a finite state coder, let $\alpha=\{1,2, \ldots, M\} \times R$ denotes the state space of MAR. The protocol functionality described by the state update function $h: G \times \alpha \rightarrow \alpha$. and the route selection function $f: G \times \alpha \rightarrow \Re$.

By our assumption, the route discovery and the route maintenance protocols may be described by $a: G \rightarrow \mathfrak{I}$ and $b: G \times \mathfrak{R} \rightarrow\{1,0\}$, where $r_{d} \in \mathfrak{R}$ denotes the disconnected route. The initial state $S_{0} \in \alpha$ is equal to $S_{0}=\left\{1, r_{d}\right\}$.

The next state is

$$
S_{n+1}=h\left(g_{n}, S_{n}\right)=\left\{\begin{array}{cc}
\left(M, a\left(g_{n}\right)\right), & S_{n}(1)=1 \|, \\
& S_{n}(2)=r_{d} \|, \\
b\left(g_{n}, S_{n}(2)\right)=0, & \text { otherwise. }
\end{array}\right.
$$

5.5. Route selection process

$$
\begin{gathered}
\qquad r_{n}=f\left(g_{n}, S_{n}\right)= \\
= \begin{cases}a\left(g_{n}\right) & S_{n}(1)=1\left\|S_{n}(2)=r_{d}\right\| b\left(g_{n}, S_{n}(2)\right)=0, \\
\left(r_{n-1}\right) & \text { otherwise. }\end{cases}
\end{gathered}
$$

As [28, Example (b)] shows MAR protocol is a finite state coder which is an example for one-sided Markov channel; by [28, Theorem 6] $\left\{R_{n}\right\}_{n=0}^{\infty}$ is AMS if $\left\{G_{n}\right\}_{n=0}^{\infty}$ is AMS, which follows directly from Definitions 4 and 5. So $\left\{R_{n}\right\}_{n=0}^{\infty}$ is AMS for any stable mobile model. 


\subsection{Link availability}

Let us consider two arbitrary nodes $v_{i}, v_{j} \in \mathfrak{R}_{S}$ and their initial positions $p_{1}$ and $p_{2}$ of these arbitrary nodes at time $t$ given by $p_{1}=\left(x_{1}, y_{1}\right)$ and $p_{2}=\left(x_{2}, y_{2}\right)$. The distance vector

$$
\begin{gathered}
\Delta d=\left|p_{1}-p_{2}\right|, \\
\Delta d=\left(\left|x_{1}-x_{2}\right|,\left|y_{1}-y_{2}\right|\right) .
\end{gathered}
$$

After traveling a small amount of time $\nabla t$, let the new positions of these nodes be $p_{1}{ }^{\prime}$ and $p_{2}{ }^{\prime}$. The distance traveled by a particular node calculated by the multiplying speed and time taken for traveling. The positions $p_{1}{ }^{\prime}$ and $p_{2}{ }^{\prime}$ after a small amount of time $\nabla t$ are defined as

$$
p_{1}^{\prime}=\left(x_{1}+\nabla t * S_{i}, y_{1}+\nabla t * S_{i}\right),
$$

and

$$
p_{2}^{\prime}=\left(x_{2}+\nabla t * S_{i}, y_{2}+\nabla t * S_{i}\right) .
$$

The new distance $\nabla d^{\prime}$ found is as follows:

$$
\begin{gathered}
\Delta d^{\prime}=\left|p_{1}^{\prime}-p_{2}^{\prime}\right|=\left|\left(\left(x_{1}+\Delta t^{*} S_{i}\right)-\left(x_{2}+\Delta t^{*} S_{i}\right)\right),\left(\left(y_{1}+\Delta t^{*} S_{i}\right)-\left(y_{2}+\Delta t^{*} S_{i}\right)\right)\right|= \\
=\left|\left(x_{1}-x_{2}\right),\left(y_{1}-y_{2}\right)\right|=\Delta d .
\end{gathered}
$$

The distance does not vary over time since MAR sets a common mobility value of all the nodes in the selected route, therefore the link availability time is high when compared to other protocols.

\section{Performance evaluation}

The following algorithms are used for comparing the performance of our proposed MAR algorithm, Lowest ID (LID) Algorithm, and Weight Based Clustering Algorithm (WBCA). LID is used as a base for most of the other algorithms and WBCA is a recent algorithm which is the modified version of WCA algorithm. The simulations are carried out using NS2 with parameters shown in Table 1.

Table 1. Simulation parameters

\begin{tabular}{|l|l|}
\hline Parameter & Values \\
\hline Simulator & NS-2 \\
\hline Transmission range & $250 \mathrm{~m}$ \\
\hline MAC layer protocol & IEEE 802.11 \\
\hline Traffic pattern & CBR \\
\hline Data packet size & 1024 bytes \\
\hline Simulation area & $1000 \times 1000 \mathrm{~m}$ \\
\hline Number of nodes & $10,20, \ldots, 50$ \\
\hline Mobility & $0-25 \mathrm{~m} / \mathrm{s}$ \\
\hline Mobility Model & random way point model \\
\hline
\end{tabular}

The following performance metrics are used to compare the proposed algorithm MAR with existing algorithms: 
1) packet delivery ratio with mobility;

2) routing overhead (bytes) with mobility;

3 ) end-to-end delay with mobility;

4) average cluster change with mobility.

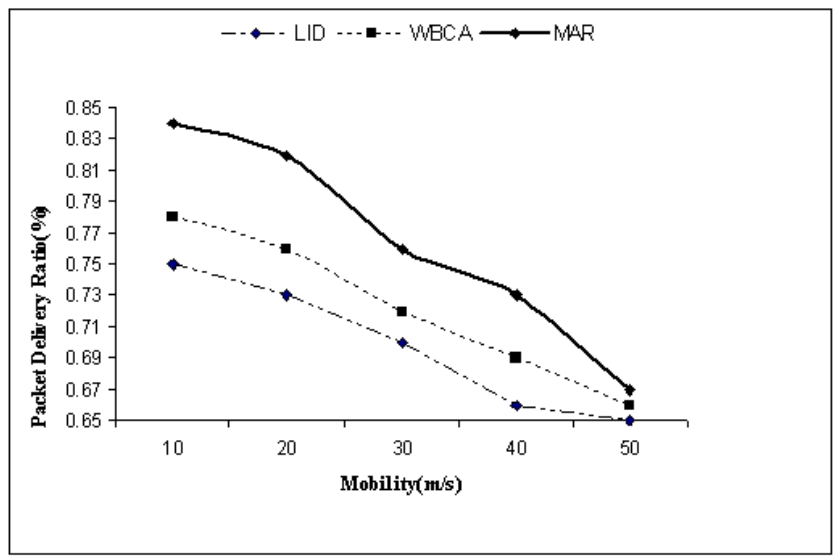

Fig. 10. Packet delivery ratio comparison

The packet delivery ratio is an important metric, since it will affect throughput of the network. When the mobility increases, the packet delivery ratio decreases due to the frequent link breaks. Fig. 10 shows the packet delivery ratio of all algorithms in our discussion. It is clear that the packet delivery ratio of our MAR algorithm is high when compared to other algorithms because the minimum mobility value of a path was found during RREQ and it is updated during RREP while establishing a path from the source to the destination.

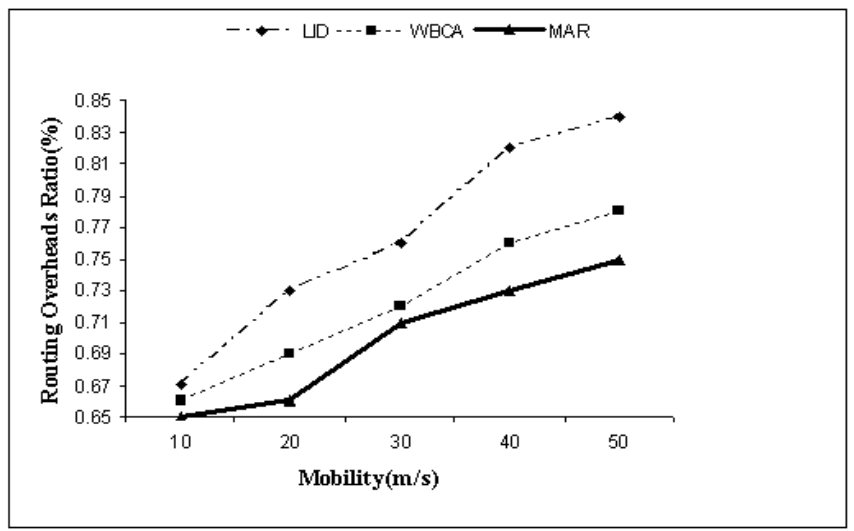

Fig. 11. Routing overheads comparison

The routing overhead is directly proportional to mobility, i.e., if the mobility increases, the routing overheads also increase because of the link break. Therefore frequent routing is required which leads to a large routing overhead. A larger number of control packets is required for the route discovery process and continuous monitoring of the paths also increases the control overheads. Fig. 11 
shows the routing overhead comparison of all three algorithms. When compared to the other algorithms, the control overheads were smaller in our MAR algorithm because the path stability was bigger. MAR path stability was high since the paths selected for data transfer have less mobility paths.

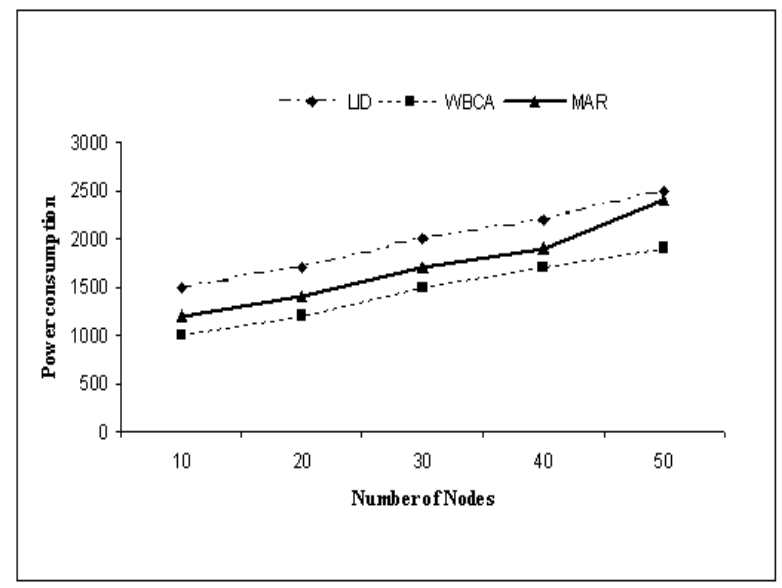

Fig. 12. Cluster head power consumption

The network density of high battery power consumption in cluster nodes is also high, due to the increased cluster members. Fig. 12 shows the power consumption by the cluster nodes demonstrated with the help of the network density. The power consumption of MAR algorithm is slightly higher than LID algorithm because of the increased path stability, achieved by adjusting the mobility of the nodes in that particular path. Therefore the energy drain rate is also high in the cluster heads.

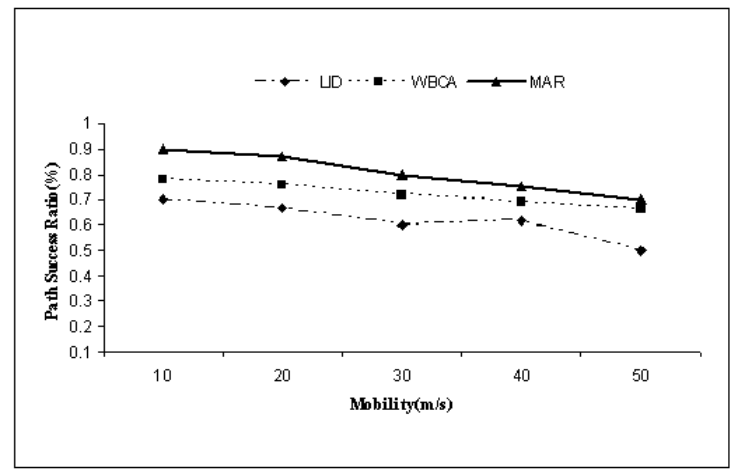

Fig. 13. Path success ratio for different mobility values

Fig. 13 shows the relative performance of three different algorithms in terms of the path success ratio for different mobility values. The path success ratio decreases when the mobility increases due to frequent link breaks. When compared to the other two algorithms, our MAR algorithm has less impact to mobility, since the mobility adjustment done in our algorithm decreases the impact of mobility to the path success ratio. 


\section{Conclusion}

In this paper MAR proposed algorithm uses the mobility and the remaining battery power as metrics to select the cluster heads. While establishing the path from a source to destination the mobility values of the intermediate node is carried by RREQ. Once RREQ reaches the destination, it carries a minimum mobility value in that particular path. During RREP, this mobility value is updated by RREP packets, therefore the path found by our algorithm is a stable path. The energy drain rate of the algorithm proposed was slightly higher because of the high utilization of the cluster heads. In future this energy drain rate will be controlled to a considerable amount.

\section{References}

1. B a s a g n i, S., M. C o n t i, S. G i or d a n o, I. S t o j m e n o v i c. Mobile Ad-Hoc Networking. Hoboken, NJ, USA, IEEE Press, Wiley Inderscience, 2004.

2. P e r k i n s, B. P. Highly Dynamic Destination-Sequenced Distance Vector Routing (DSDV) for Mobile Computers. - In: Proceedings of ACM SIGCOMM'94,1994, 234-244.

3. C 1 a u s e n, T., P. J a c q u e t. Optimized Link State Routing Protocol (OLSR). - In: IETF Mobile Ad-Hoc Networking Working Group INTERNETDRAFT, CF3626, October 2003. http://www.ietf.org/rfc/rfc3626.txt

4. P e r k i n s, C., E. B e $1 \mathrm{~d}$ i n g-R o y e r, S. D a s. Ad-Hoc On-Demand Distance Vector (AODV) Routing. - In: IETF Mobile Ad-Hoc Networking Working Group INTERNETDRAFT, RFC3561, July 2003. http://www.ietf.org/rfc/rfc3561.txt

5. J o h n s o n, D., Y. H u, D. M a $1 \mathrm{t}$ z. The Dynamic Source Routing Protocol (DSR). - In: IETF Mobile Ad-Hoc Networking Working Group INTERNET DRAFT, RFC 4728, February 2007. http://www.ietf.org/rfc/rfc4728.txt.

6. P e a r $1 \mathrm{~m}$ a n, M. R., Z. J. H a a s. Determining the Optimal Configuration for the Zone Routing Protocol. - IEEE Journal on Selected Areas in Communications, Vol. 17, 1999, Issue 8, $1395-1414$.

7. S a m a r, P., M. R. P e a r $1 \mathrm{~m}$ a n, Z. J. H a a s. Independent Zone Routing: An Adaptive Hybrid Routing Framework for Ad-Hoc Wireless Networks. - IEEE/ACM Transactions on Networking, August 2004.

8. M u r t h y, C. S. R., B. S. M a n o j. Ad-Hoc Wireless Networks: Architectures and Protocols. New Jersey, Prentice Hall PTR, 2004.

9. B a k e r, D. J., A. E p h r e $\mathrm{m}$ i d e s. The Architectural Organization of a Mobile Radio Network via a Distributed Algorithm. - IEEE Transactions on Communications, Vol. 29, 1981, No 11, 1694-1701.

10. P a r e k h, A. K. Selecting Routers in Ad-Hoc Wireless Network. - In: Proceedings of the SBT/IEEE International Telecommunication Symposium (ITS'94), 1994

11. G e r 1 a, M., J. T.C. T s a i. Multicluster, Mobile, Multimedia Radio Network. - Wireless Networks, Vol. 1, 1995, No 3, 255-265.

12. $\mathrm{S}$ h a r m a, A n j u, S h in i A g a r w a 1 , R a vin d r a S i n g h R a th or e. Cluster Based Routing in Mobile Ad-Hoc Wireless Networks Using Neuro-Genetic Paradigm. International Journal of Scientific \& Engineering Research, Vol. 3, July 2012, Issue 7.

13. G o m a t h i, M., G. Raj e ndran. Fuzzy Cost Enabled Cluster Based Multipath Routing Algorithm for Mobile Ad-Hoc Networks. - Journal of Computer Science, Vol. 8, 2012, Issue 9, 1434-1440. 
14. E p h r e m i d e s, A., J. E. W I e s e $1 \mathrm{t} \mathrm{h} \mathrm{I} \mathrm{e} \mathrm{r,} \mathrm{D.} \mathrm{J.} \mathrm{B} \mathrm{a} \mathrm{k} \mathrm{e} \mathrm{r.} \mathrm{A} \mathrm{Design}$ Concept for Reliable Mobile Radio Networks with Frequency Hopping Signaling. Proceedings of IEEE, Vol. 75, 1987, No 1, 56-73.

15. B a k e r, D. J., A. E p h r e m i d e s. The Architectural Organization of a Mobile Radio Network via a Distributed Algorithm. - IEEE Transactions on Communications, Vol. 29, 1981, No 11, 1694-1701.

16. B a s u, P., N. K h a n, T. D. C. L i t t 1 e. A Mobility Based Metric for Clustering in Mobile Ad-Hoc Networks. - In: Proceedings of IEEE Workshop on Wireless Networks and Mobile Computing (ICDCS'01), Phoenix, Ariz, USA, 2001.

17. B a s a g n i, S. Distributed Clustering for Ad-Hoc Networks. - In: Proceedings of the International Symposium on Parallel Architectures, Algorithms and Networks (I-SPAN'99), 1999, 310-315.

18. $\mathrm{G} \mathrm{h}$ o $\mathrm{s} \mathrm{h}$, R., S. B a s a g n i. Limiting the Impact of Mobility on Ad-Hoc Clustering. - In: Proceedings of 2nd ACM International Workshop on Performance Evaluation of Wireless Ad-Hoc, Sensor, and Ubiquitous Networks (PE-WASUN'05), Montreal, Canada, October 2005, 197-204.

19. D a s, S., M. C h a t t e r j e e, D. T u r g u t. WCA: A Weighted Clustering Algorithm for Mobile Ad-Hoc Networks. - Journal of Cluster Computing, Vol. 5, 2002, No 2, 193-204.

20. R a m a $n$ a t h a n, R., R. R o s a 1 e s h a i n. Topology Control of Multihop Wireless Networks Using Transmit Power Adjustment. - In: Proceedings of 19th Annual Joint Conference of the IEEE Computer and Communications Societies (INFO-COM'00), 2000, 404-413.

21. P a u l, K., S. B a n d y o p a d h y a y. Self-Adjusting Transmission Range Control of Mobile Hosts in Ad-Hoc Wireless Networks for Stable Communication. - In: Proceedings of 6th International Conference on High Performance Computing (HiPC'99), Kolkata, India, 1999.

22. $\mathrm{Ch}$ in a ra, S u ch is mit a, S a n t a n u k m a r R a th. Topology Control by Transmission Range Adjustment Protocol for Clustered Mobile Ad-Hoc Networks. - International Scholarly Research Network ISRN Communications and Networking Volume Article, ID 147925. 10 pages. doi:10.5402/2011/147925, 2011.

23. Y o o n, J., M. L i u, B. N o b 1 e. Random Waypoint Considered Harmful. - In: INFOCOM: 22nd Annual Joint Conference of the IEEE Computer and Communications Societies, March 2003, 1312-1321.

24. Y o o n, J., M. L I u, B. N o b 1 e. Sound Mobility Models. - In: Proceedings IEEE International Symposium on Mobile Ad-Hoc Networking and Computing, MobiHoc, September 2003, 205-216.

25. N a v i d i, W., T. C a m p. Stationary Distributions for the Random Waypoint Mobility Model. IEEE Trans. Mobile Comput., Vol. 3, 2004, No 1, 99-108.

26. B e t t s t e t t e r, C., H. H a r t e n s t e i n, X. P e r e z-C o s t a. Stochastic Properties of the Random Waypoint Mobility Model. ACM/Kluwer Wireless Networks, 2004.

27. B o u d e c, J. On the Stationary Distribution and Location of Random Waypoint. - IEEE Trans. Mobile Comput., Vol. 4, July/August 2005, No 4, 404-406.

28. B o u d e c, J., M. V o j n o v i'c. The Random Trip Model: Stability, Stationary Regime, and Perfect Simulation. - IEEE/ACM Trans. Networking, Vol. 14, December 2006, No 6, $1153-1166$.

29. T i m o, R., K. B 1 a c k m o r e, L. H a n 1 e n. Strong Stochastic Stability for MANET Mobility Models. - In: Tech. Rep. PA006222, NICTA, May 2007, 15th IEEE International Conférence On Networks (ICON), 2007.

30. K i e f f e r, J., M. R a h e. Markov Channels are Asymptotically Mean Stationary. - SIAM Journal Mathematical Analysis (SIMA), Vol. 12, May 1981, No 3, 293-305.

31. G r a y, R., J. K i e f f e r. Asymptotically Mean Stationary Measures. - Journal of Annals of Probability, Vol. 8, October 1980, No 5, 962-973.

32. T i m o, R., K. B l a c k m o r e, L. H a n 1 e n. Strong Stochastic Stability for MANET Mobility Models. - In: Tech. Rep. PA006222, NICTA, May 2007, 15th IEEE International Conférence on Networks (ICON), 2007. 